\title{
Prevalence and Risk Factors of Pregnancy Induced Hypertension Including Preeclampsia/Eclampsia in Women of Odisha, an Eastern Indian State
}

\section{Madhusmita Bal ${ }^{1}$, Sujata Dixit ${ }^{1}$, Subrata K Rath ${ }^{2}$, Tahziba Hussain ${ }^{1}$, Nihar Ranjan Nayak ${ }^{3}$, Sanghamitra Pati ${ }^{1}$ and Manoranjan Ranjit ${ }^{1 *}$}

${ }^{1}$ ICMR-Regional Medical Research Centre, Bhubaneswar, Odisha, India

${ }^{2}$ Department of Obstetrics and Gynaecology, KIMS, KIIT University, Bhubaneswar,

Odisha, India

${ }^{3}$ Department of Obstetrics and Gynecology Wayane State University School of

Medicine, USA

*Corresponding Author: Manoranjan Ranjit, Scientist-G, ICMR-Regional Medical

Research Centre, Bhubaneswar, Odisha, India.
Received: February 16, 2021

Published: April 12, 2021

(C) All rights are reserved by Manoranjan

Ranjit., et al.

\begin{abstract}
Aim: Despite several measures the maternal mortality rate (MMR) and infant mortality rate (IMR) in the state of Odisha is significantly high. Since pregnancy induced hypertension (PIH) including pre-eclampsia or eclampsia (PE or E) is one of the leading causes of maternal and fetal mortality/morbidity, we attempted to analyze the burden and risk factors of PIH including PE/E for the first time among pregnant women in Odisha.

Methods: The medical records of 2393 mothers and their infants were retrospectively audited from January 2015 to December 2016 in KIMS Hospital, Bhubaneswar, the capital city of the state.

Results: Out of total 150 (6.2\%) cases presenting pregnancy induced hypertension disorder (PIHD), 105 (70\%) had PIH, 32 (30.47\%) had developed PE and 13 (12.38\%) eclampsia. Of the babies born to mothers with PIH, 56\% had low birth weight (<2.5kg) and 6.6\% (10 out of 150 live births) had died during their infancy. Oedema, pallor, pain in lower abdomen, headache and vaginal leaking were the most common clinical features, while chronic hypertension, gestational diabetes, PIH in previous pregnancy and family history of hypertension were the highest risk factors of PE/E.

Conclusions: The present study highlights the magnitude/associated risk factors of the PIHD and underscores the need for a large scale community survey to find out the exact burden of the disease for development of strategy for proper management of pregnant women during the time of antenatal checkups in the state that contributes high MMR and IMR in the country.
\end{abstract}

Keywords: Eclampsia; India; PIH; Prevalence; Pre-eclampsia; Risk Factors

\section{Introduction}

Pregnancy induced hypertension (PIH), a condition specific to pregnancy, includes gestational hypertension, pre-eclampsia (PE) and eclampsia (E), which is associated with maternal mortality, premature delivery, intra-uterine growth retardation (IUGR), abruptio placentae and intra-uterine death $[1,2]$. PIH has remained a significant public health threat in both developed and developing countries that contribute to maternal and perinatal morbidity and mortality. Further, studies have shown that a woman's lifetime risk of dying from pregnancy-induced complications in developing countries is 14 times higher than in developed countries [3]. According to an estimate pregnancy induced hypertension affects about 5 - 8\% of all pregnant women worldwide and pre-eclampsia occurs in $10 \%$ of all pregnancies, the second leading cause of direct maternal and fetal deaths [4]. But Asian women with preeclampsia have been observed with worse pregnancy outcomes than others [5]. 
Maternal mortality is one of the indices used to evaluate a country's health care delivery and country's economical developmental stage [6]. Further reduction of maternal deaths is a high priority for the international community, especially in view of increased attention on the Millennium Development Goals (MDGs). MDGs created goals for maternal and child health that requires countries to have reduced by 2015 maternal mortality ratio by $75 \%$ compared to that of 1990. However according to recent data the MMR/IMR (235/51) in Odisha is very high compared to the national level (178/40) (SRS 2010-12 and SRS 2013) [7] warranting an in-depth study. According to a recent report based on nationwide survey the prevalence of preeclampsia in India is 8-10\% [8]. Numerous risk factors for pre-eclampsia have been suggested but only some have actually been established in multivariate models that permit simultaneous control for possible confounders. As suggested, ethnicity could be a risk factor for developing preeclampsia because of difference in the prevalence of in different populations and different ethnic groups $[9,10]$. Since the population of Odisha is genetically heterogeneous [11] and no clear information on the prevalence and risk factors of $\mathrm{PIH}$ is known, there is a need for obtaining such information in order to generate evidences for effective decision making to prevent and control the disease. Keeping in view of this the present study was conducted to explore the prevalence and risk factors of PIH including PE/E in pregnant women of Odisha.

\section{Materials and Methods}

The present retrospective study was carried out in Kalinga Institute of Medical Sciences (KIMS) Hospital, (KIIT University), Bhubaneswar, Odisha. Being a leading medical institute in the state it provides comprehensive obstetric care for the people of Odisha and neighboring states. It also offers teaching and training to both medical under graduate and post graduates. The present hospital based retrospective study was conducted for the period $1^{\text {st }}$ January 2015 to $31^{\text {st }}$ December 2016. A total number of 4056 pregnant women registered and/or admitted to the Obstratic and Gynecology Department of KIMS Hospital, Bhubaneswar for antenatal check up and/or delivery during this period. The details of the pregnant women were extracted from the medical record department of KIMS Hospital. Every medical chart was reviewed to collect the data. The pregnant women with a gestational age of 20 weeks or greater were included in the study. Pregnant women's gestational age was measured based on women recall of the last menstrual period (LMP). Exclusion criteria were: women with multiple pregnancies, women with proteinuria at less than 20 weeks' gestation and pregnancies complicated by foetal malformations. The demographic data, obstetric history, gestational age of delivery, blood pressure, infant weight and sex, maternal and neonatal outcomes were collected in the pretested format.

PIH was defined as those pregnant women with hypertension [systolic blood pressure (SBP) $\geq 140 \mathrm{mmHg}$ and/or diastolic blood pressure (DBP) $\geq 90 \mathrm{mmHg}$ ] at or after 20 weeks gestation without proteinuria, where as pre-eclampsia is defined as the presence of proteinuria along with symptoms of PIH. The eclampsia is defined as new onset of generalized tonic-clonic seizure and/or unexplained coma during pregnancy or postpartum in a woman with signs or symptoms of preeclampsia. Blood pressure readings were taken while the woman was seated in the upright position using a mercury sphygmomanometer apparatus which covers two-thirds of the upper arm. Proteinuria was assessed using urine dipstick method and was part of the routine investigation for all pregnant women. Those women having protein level of $1+$ classified as having proteinuria.Low birth weight was defined as weight of newborns below $2.5 \mathrm{~kg}$ at term or below tenth percentile for the gestational age. As per WHO Preterm birth was defined as birth below 37 weeks gestation.

Our study being based on retrospective analysis of data in women who received standard management at the hospitals do not need approval of institute's ethics committee. Communication with the hospital administrators were made through formal letter and the purpose of the study has been informed and confidentiality of the patient has been maintained.

The quantitative data were entered and analyzed using the SPSS version 16.0 (SPSS Inc.) to generate frequencies, means and odds ratios. P-value of 0.05 was set as the significance level.

\section{Results}

During the study period ( $1^{\text {st }}$ January 2015 to $31^{\text {st }}$ December 2016), a total number of 4056 women were registered for ANC and out of them 2393 cases filled up the criteria for inclusion in the present study. Out of them 150 women were diagnosed to have PIHD thus the prevalence was 6.26\% (150 out of 2393). Those with PIHD were older than those without PIHD (median age 30 and 26 respectively, $\mathrm{p}=0.0009$ ). Out of the total PIHD cases included, $70.0 \%$ (105 out of 150 ) continued to be hypertensive (PIH), 30.47 (32 out of 105) pre-eclampsia and $12.38 \%$ (13 out of 105) eclampsia. Of the babies born to mothers with PIHD 56.0\% (84 out of 150) had low birth weight compared to 9.3\% (209 out of 2243) among those born without PIHD. Of those women who had PIHD, 52.7\% (79 out of 150) delivered their babies before 37 weeks of gestation where as only $10.7 \%$ (241 out of 2243) was seen in case of women without PHID ( $\mathrm{n}=2243$ ). The delivery outcome of each group of patients has been depicted in table 1 . 
Prevalence and Risk Factors of Pregnancy Induced Hypertension Including Preeclampsia/Eclampsia in Women of Odisha, an Eastern Indian State

\begin{tabular}{|l|c|c|c|c|}
\hline Variables & $\begin{array}{c}\text { PIH } \\
(\mathbf{n = 1 0 5})\end{array}$ & $\begin{array}{c}\text { Pre-eclampsia } \\
(\mathbf{n = 3 2})\end{array}$ & Eclampsia (n = 13) & P value \\
\hline Mean Age (Years) & $27.50 \pm 4.44$ & $25.93 \pm 3.37$ & $24 \pm 3.65$ & 0.007 \\
\hline Systolic Blood Pressure (mmHg) & $154.09 \pm 16.38$ & $159.46 \pm 19.32$ & $162.76 \pm 28.39$ & 0.134 \\
\hline Diastolic Blood Pressure (mmHg) & $93.62 \pm 11.45$ & $105.8 \pm 12.45$ & $106.92 \pm 13.77$ & $<0.001$ \\
\hline Mean Pulse rate & $88 \pm 24.83$ & $96.85 \pm 25.66$ & $93.66 \pm 10.82$ & 0.173 \\
\hline Mean Gestational age (Weeks) of delivery & $35.78 \pm 3.52$ & $34.53 \pm 4.30$ & $32.20 \pm 4.09$ & 0.003 \\
\hline Intra Uterine Growth Retardation & 5 & 15 & 6 & $<0.001$ \\
\hline Birth Weight (kg) & $2.48 \pm 0.70$ & $2.04 \pm 0.67$ & $1.73 \pm 0.36$ & $<0.001$ \\
\hline Infant Death & 2 & 3 & 5 & P $<0.001$ \\
\hline Sex of Infant & & & & 0.008 \\
Male & 56 & 19 & 6 & 7 \\
Female & 49 & 13 & & \\
\hline
\end{tabular}

Table 1: Clinical and Delivery outcome variables among the pregnant women with PIH and Preeclamsia/Eclampsia.

Out of 150 mothers with PIHD one hundred twenty three (82\%) delivered by caesarean section (CS) and 27 (18\%)-had an assisted delivery. The major reasons for caesarean section delivery were previous CS, macrosomia, breech presentation and slow progress. Intra uterine growth retardation was observed in 26 cases out of which 21 belong to pre-eclampsia/eclampsia and 5 belongs to PIH group. Women who were multigravida (55 out of $150,36.6 \%$ ) were less likely to develop PIH compared to primagravid (95 out of 150, $63.3 \%$ ) and the association was statistically significant (OR 2.04, $\mathrm{p}<0.0001$ ). No difference in the sex of the infant was observed between the PIH and PE/E groups. Significantly high infant mortality was found in PE/E group (8 out of $45,18 \%$ ) compared to cases with only PIH (2 out of 105, 1.9\%). No significant difference was observed regarding systolic pressure and mean pulse rate among the three groups (PIH, pre-eclampsia and eclampsia). However, diastolic pressure was significantly higher $(\mathrm{P}<0.0001)$ in pre-eclampsia and eclampsia cases compared to PIH cases (Table 1). The most common symptoms experienced was headache (18\%) vomiting (12\%), epigastric pain (8\%), chest pain or dyspnoea (2\%), back pain $(2.7 \%)$, blurring of vision (3.3\%) and HELP syndrome (1.3\%). Oedema was present in 88 (58.7\%), pain in lower abdomen in 44 (29.3\%). Fluid leaking from the vagina suggesting premature rupture of membrane was present in 21 (14\%) of the PIH cases. Loss of foetal movement was observed in $2.7 \%$ and oligohydrominos in $2 \%$ of pregnancy with PIH (Figure 1). Nifedipine and Labetelol was the drug of choice for management of PIHD.

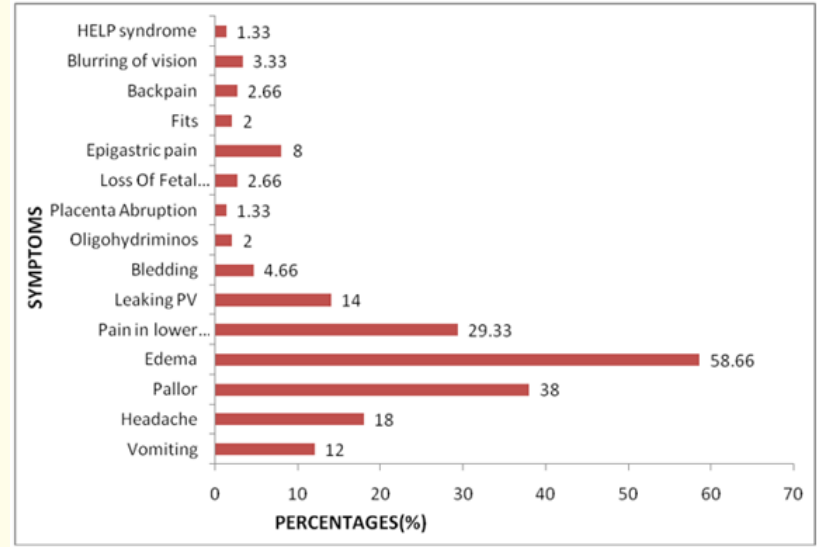

Figure 1: Clinical features among the pregnant women with pregnancy induced hypertension and preeclampsia/eclampsia.

Out of 150 PIHD cases, 111 mothers had other systemic diseases. These were chronic hypertension (diastolic $>80 \mathrm{~mm} \mathrm{Hg}$ ) at booking (31.42\%), hypothyroidism (26.66\%) and gestational diabetes $(4.76 \%)$ and/or family history of chronic diseases (family diabetic: $16.19 \%$, family hypertension: $11.42 \%$ and family hypothyroidism: $3.80 \%$ ). While, at least $40 \%$ of women having pre-eclampsia and $61.5 \%$ of women having eclampsia were either having chronic disorders or having family history of chronic diseases. On 
Prevalence and Risk Factors of Pregnancy Induced Hypertension Including Preeclampsia/Eclampsia in Women of Odisha, an Eastern Indian State

analysis it is evident that women with chronic hypertension, gestational diabetes, PIH in previous pregnancy and family history of hypertension had greater chance of developing pre-eclampsia and eclampsia among the PIH cases $(\mathrm{P}<0.0001)$ (Table 2).

\begin{tabular}{|c|c|c|c|c|c|c|}
\hline Symptoms & $\begin{array}{l}\text { Total No } \\
\text { of Cases }\end{array}$ & PIH & Preeclampsia & Eclampsia & Odds Ratio & $95 \%$ CI \\
\hline Diabetics & 9 & 8 & 0 & 1 & 0.2756 & 0.0334 to 2.2711 \\
\hline Hypothyroidism & 28 & 22 & 4 & 2 & 0.5804 & 0.2179 to 1.5459 \\
\hline Gestational Diabetics & 5 & 1 & 3 & 1 & 10.147 & 1.1009 to 93.5099 \\
\hline Chronic Hypertension & 33 & 21 & 9 & 3 & 1.4545 & 0.6434 to 3.2881 \\
\hline PIH in early pregnancy & 7 & 2 & 3 & 2 & 6.4375 & $1.1998-34.5414$ \\
\hline Family Diabetic & 17 & 15 & 1 & 1 & 0.2791 & 0.0611 to 1.2754 \\
\hline Family Hypertension & 12 & 4 & 5 & 3 & 5.4595 & 1.5518 to 19.2077 \\
\hline Family Hypothyroidism & 2 & 0 & 2 & 0 & 0.0 & 0.0 \\
\hline
\end{tabular}

Table 2: Personal and family history of chronic diseases among the pregnant women with PIH and PE/E.

\section{Discussion}

Pregnancy induced hypertension is a serious and poorly understood complication of pregnancy, which can progress to pre-eclampsia and eclampsia, is an important cause of maternal mortality in developing countries [12]. It also contributes to morbidities (acute and long-term), perinatal deaths, preterm birth, and intrauterine growth retardation [13]. The prevalence of hypertensive disorder in pregnancy varies according to geographic regions of world and ranges from $1.5 \%-7.5 \%$ [3]. We evaluated a large study population over a two-year period in a large maternity centre in Bhubaneswar for the prevalence of PIH including pre-eclampsia and eclampsia. This is one of the large maternity centers in Bhubaneswar dealing with over 2500 deliveries a year. Our data shows the prevalence of PIHD to be $6.2 \%$ in Bhubaneswar, an eastern Indian city similar with a study done at Pune, situated in western part of India [8]. However, it is lower than the findings of studies conducted in Ethiopia (7.6\%), Birmingham (11.9\%), and Nigeria (16\%) [14-16]. The variations can be attributed to racial differences, socioeconomic status and some other parameters like parity and age. In India, the incidence of preeclampsia is reported to be $8-10 \%$ and eclampsia in $0.71 \%$ of the pregnancies [8]. But we have observed PIH $4.4 \%$ (105 out of 2393), preeclampsia in 1.3\% (32 out of 2393 pregnancies) and eclampsia in $0.5 \%$ (13 out of 2393 pregnancies) of the study population. The variation of pre-eclampsia/eclampsia prevalence not only due to geographic differences and study design, but may also be attributed to differences in diagnostic capacities or ac- cessibility of services [17]. Some workers may have used PIH and PE interchangeably. Moreover, population based study and hospital based study would yield different prevalence. One of the reasons for low incidence of preeclampsia/eclampsia in our study might be that our study population is a small section of the total population (woman with affluent economical background) those primarily seeks antenatal care at tertiary care facility/corporate hospitals and hence smaller local/regional studies may be conducted at different parts of the state in different population to estimate the real burden of PIHD.

Age has an important influence on the incidence of hypertensive disorders of pregnancy. In our study highest incidence of the hypertensive disorders occurred among those aged 30 years. This could be because the majority of conceptions take place in this age group in our country. But other studies showed that young primigravidae under 20 years and all patients over 30 years have an increased chance of hypertension [18]. In our study majority of pre-eclampsia and eclampsia were apparently higher in younger pregnant women (less than 30 years) in contrast to other studies which showed pre-eclampsia is more frequent in patients younger than 21 years of age and in older than 35 . One explanation can be that, our study population being urban based there is delay in the age of marriage and exercise of reproductive choice.

We have got a significant difference in the mean age of years among the three groups' i.e. PIH, pre-eclampsia and eclampsia. 
However eclampsia was observed in women having lower age group which is supported by other studies [17]. Though there is no significant difference in systolic blood pressure which may be due to small sample size still a significant high level of diastolic pressure was observed in women having pre-eclampsia/eclampsia. Mean gestational age was lower in pre-eclampsia and eclampsia cases because delivery is recommended for all women with severe pre-eclampsia no later than 34 weeks gestation [13]. However delivery is typically recommended for women who develop preeclampsia, regardless of disease severity [19]. The birth weight of the infant was significantly lower in pre-eeclmpsia and eclampsia cases as compared to PIH cases and cases without PIH. Similarly higher rate of infant death was observed in pre-eclampsia and eclampsia cases as compared to PIH and non PIH cases. These findings support previous findings that pre-eclampsia and eclampsia poses higher risk for perinatal death, preterm birth and low birth weight [17]. A population-based study from the British Columbia Perinatal Database found that neonatal mortality (deaths among infants 0-27 days' chronological age) and infant mortality (death among infants 0-364 days' chronological age) was 5.5 and 3.5 times greater in the late-preterm group, respectively [20].

The women having PIH, pre-eclampsia and eclampsia showed a number of features but oedema, pallor, pain in lower abdomen were the most common clinical features among these cases. Besides this severe headache, blurry vision, epigastric pain, signs and symptoms of liver disease (nausea and/or vomiting with abdominal pain), rise in liver enzymes, HELP syndrome and also high blood pressure were observed among the study population.

Women having family history of hypertension had more chance of developing hypertensive disorder in our population. This finding is at par with the findings in pregnant women of Brazil, Sudan, Uganda and Thailand [21-24]. This warrants studies on genetic factors responsible for predisposing women to an increase risk of preeclampsia. Our study also reveals that women with hypertension before pregnancy, PIH in previous pregnancy and gestational diabetes have higher chance of developing PIH. This was in concordance with the researches done in France, Nigeria and Northeast Ethiopia $[16,25,26]$. Since all the above risk factors can be identified during antenatal check-ups, risk screening and the referral of higher-risk pregnancies for more intensive care at the check-up stage could help reduce the incidence of pre-eclampsia/eclampsia or its adverse outcomes. Ongoing evidence suggests that various adult disease states (hypertension, obesity, diabetes) may begin during foetal development, and the insults from pre-eclampsia exposure accrued during sensitive periods of development may predispose an individual to an increased risk of disease in adulthood. This is supported by the epidemiological investigation that showed infants exposed to pre-eclampsia during gestation are associated with an increased risk of diabetes and cardiovascular morbidity in adulthood [27], thereby posing a daunting challenge within the context of double burden and limited resources in the developing world [28]. This study has several limitations. First, being a hospital-based record analysis, the findings may not be generalizable to the broader population, especially in settings where institutional delivery rates are low. Second, there are potential biases and errors arising from the dataset. Because the study was not originally designed to detect PIH, pre-eclampsia/eclampsia and its outcomes or to screen for risk factors, under diagnoses or likelier detection of more clinically evident cases could have transpired. Lastly, infant mortality in our study only includes intra hospital deaths, and the inability to capture post-discharge mortality implies that our results could be underestimated. Interpretation of results should thus consider all the above limitations.

\section{Conclusion}

The present study reveals the incidence of pre-eclampsia/eclampsia to be $30 \%$ among the pregnant women having hypertensive disorder of pregnancy and is associated with low birth weight and infant death. So it needs to be prioritised in reducing maternal and infant mortality in this state reporting high MMR/IMR. Chronic hypertension, gestational diabetes, family history of hypertension and PIH in earlier pregnancy, was the highest risk factors for developing PE/E. Therefore precise blood pressure measurement before and during pregnancy can serve as a good indicator of pregnancy outcome. However large cross sectional study may be undertaken to estimate the exact burden of hypertensive disorders during pregnancy and their consequences so as to develop an appropriate strategy for prevention and timely management of the disorders at antenatal care visits. Implementation of effective interventions like pre conception counselling of pregnant women's health seeking behaviour would provide a chance to diagnose preeclampsia as early as possible that improve maternal and child health status.

\section{Acknowledgements}

The authors wish to acknowledge the Director, ICMR-Regional Medical Research Centre, Bhubaneswar and Medical Superinten- 
dent, Kalinga Institute of Medical Sciences (KIMS), KIIT University, Bhubaneswar for taking interest and providing all the necessary facilities to conduct this research. Help rendered by the staff of the medical record room of KIMS Hospital during retrieval of the medical record of the patients is thankfully acknowledged

\section{Conflict of Interest}

The author(s) declared no potential conflicts of interest with respect to the research, authorship, and/or publication of this article.

\section{Bibliography}

1. Beltran AJ., et al. "Associations of Meteorology with Adverse Pregnancy Outcomes: A Systematic Review of Preeclampsia, Preterm Birth and Birth Weight". International Journal of Environmental Research and Public Health 11 (2014): 91-172.

2. Kharaghani R., et al. "Prevalence of preeclampsia and eclampsia in Iran”. Archives of Iranian Medicine 19 (2016): 64-71.

3. WHO, UNICEF, UNFPA, The World Bank, UN Population Division. Trends in maternal mortality: 1990 to 2013. In: WHO, UNICEF, UNFPA, The World Bank, UN Population Division (2013).

4. Atallah AN., et al. "Calcium supplementation during pregnancy for preventing hypertensive disorders and related problems". Cochrane Database System Review 19.3 (2006): CD001059.

5. Cripe S., et al. "Perinatal Outcomes of Southeast Asians with Pregnancies Complicated by Gestational Diabetes Mellitus or Preeclampsia". Journal of Immigrant and Minority Health 14 (2012): 747-753.

6. Buga GAB., et al. "Pre-elampsia predisposes for hypertensive Diseases in next pregnancy". East African Medical Journal 76.4 (1999): 191-198.

7. Office of the Registrar General, India, Ministry of Home Affairs, Govt. of India, Sample Registration System (SRS) Statistical Report: Maternal and Infant Mortality (2013).

8. Sajith M., et al. "Incidence of pregnancy induced hypertension and prescription pattern of antihypertensive drugs in pregnancy". International Journal of Pharmaceutical Sciences and Research 5.4 (2014): 163-170.

9. Roberts JM., et al. "Recent insights into the pathogenesis of pre-eclampsia”. Placenta 23 (2002): 359-372.
10. Xiao J., et al. "Is ethnicity a risk factor for developing preeclampsia? An analysis of the prevalence of preeclampsia in China". Journal of Human Hypertension 28 (2014): 694-698.

11. Sahoo S., et al. "Influence of language and ancestry on genetic structure of contiguous populations: A microsatellite based study on populations of Orissa". BMC Genetics 6 (2005): 4.

12. Cisse CT., et al. "Preeclampsia: current aspects of physiopathology, clinic and treatment". Dakar Medica 49.3 (2004): 152161.

13. Sibai B. "Pre-eclampsia”. Lancet 365.9461 (2005): 785-799.

14. Wolde Z., et al. "Hypertensive disorders of pregnancy in Jimma University specialized hospital". Ethiopian Journal of Health Sciences 21.3 (2011): 47.

15. Direkvand-Moghadam A., et al. "Predictive factors for preeclampsia in pregnant women: aunvariate and multivariate logistic regression analysis". Acta Biochimica Polonica 59.4 (2012): 673-677.

16. Guerrier G., et al. "Factors associated with severe preeclampsia andeclampsia in Jahun, Nigeria". International Journal of Womens Health 5 (2013): 509-513.

17. Bilano VL., et al. "Risk Factors of Pre-Eclampsia/Eclampsia and Its Adverse Outcomes in Low- and Middle-Income Countries: A WHO Secondary Analysis". PLoS ONE 9.3 (2014): 91198.

18. Zibaeenazhad MJ., et al. "The prevalence of hypertensive disorders of pregnancy in Shiraz, Southern Iran". Iranian Cardiovascular Research Journal 4 (2010): 169-172.

19. Barton JR., et al. "Mild gestational hypertension remote from term: progression and outcome". American Journal of Obstetrics and Gynecology 184.5 (2001): 979-983.

20. Khashu M., et al. "Perinatal outcomes associated with preterm birth at 33 to 36weeks' gestation: a population-based cohort study". Pediatrics 123.1 (2009): 109-113.

21. Dalmaz CA., et al. "Risk factors for hypertensive disorders of pregnancy in southern Brazil". Revista da Associacao Medica Brasileira 57.6 (2011): 692-696.

22. Adam I., et al. "Malaria and pre-eclampsia in an area with unstable malaria transmission in Central Sudan". Malaria Journal 10 (2011): 258. 
23. Kiondo P., et al. "Risk factors for pre-eclampsia in Mulago Hospital, Kampala, Uganda". Tropical Medicine and International Health 17.4 (2012): 480-487.

24. Aksornphusitaphong A., et al. "Risk factors of early and late onset pre-eclampsia". Journal of Obstetrics and Gynecology Research 39.3 (2013): 627-631.

25. Lecarpentier E., et al. "Risk factors of superimposed preeclampsia in women with essential chronic hypertension treated before pregnancy”. PLOS One 8.5 (2013): 62140.

26. Tessema GA., et al "Preeclampsia and associated factors among pregnant women attending antenatal care in Dessie referral hospital, Northeast Ethiopia: a hospital-based study". BMC Pregnancy and Childbirth 15 (2015): 73.

27. Simmons RA. "Developmental origins of adult disease". Pediatric Clinics of North America 56 (2009): 449-466.

28. Bygbjerg IC. "Double burden of noncommunicable and infectious diseasesin developing countries". Science 337 (2012): 1499-1501.

\section{Assets from publication with us}

- Prompt Acknowledgement after receiving the article

- Thorough Double blinded peer review

- Rapid Publication

- Issue of Publication Certificate

- High visibility of your Published work

Website: www.actascientific.com/

Submit Article: www.actascientific.com/submission.php

Email us: editor@actascientific.com

Contact us: +919182824667 